\title{
Response to letter to the editor: "Impact of intraoperative stimulation mapping on high-grade glioma surgery outcome: a meta-analysis"
}

\author{
Jasper K.W. Gerritsen ${ }^{1}$ (D) - Arnaud J.P.E. Vincent ${ }^{1}$
}

Received: 5 December 2019 / Accepted: 10 December 2019

(C) Springer-Verlag GmbH Austria, part of Springer Nature 2019

\section{Dear Editor,}

We would like to thank Dr. Giussani and Dr. Di Cristofori and their colleagues at the Gerardo Hospital (Monza, Italy) for their interest in our paper, and we would like to clarify in this answer certain aspects of our paper as well as elaborate on our thoughts regarding the use of awake craniotomy (AC) in glioblastoma (GBM) surgery.

In their letter to the editor, Dr. Giussani and Dr. Di Cristofori note potential concerns with the methodology and inclusion criteria of the studies in our meta-analysis. We agree that the absence of data on molecular markers such as MGMT status or IDH wt $/ \mathrm{mt}$ status (the authors also mentioned the $1 \mathrm{p} 19 \mathrm{q}$ codeletion which in our opinion is in this GBM population is of less interest) is a limitation of our study. Even though we acknowledge the importance of molecular markers in the analysis of GBM (sub)groups, we unfortunately were not able to include this in our analysis and paper because this information was not consistently mentioned in the specific literature we found for this systematic review.

We agree with Dr. Giussani and Dr. Di Cristofori that the time span of the included studies is quite wide. We did this on purpose to present an overview of the overall evidence regarding the use of intraoperative stimulation mapping (ISM) in GBM surgery to its full extent. However, we included only a few papers from the pre-Stupp era (which we define as papers published in 2005 or earlier), which — as stated by the earlier mentioned authors - could have been a bias in the overall survival analysis. Looking at the data published in our Data

No portions of the contents of this paper have been presented nor published previously.

This article is part of the Topical Collection on Brain Tumors

Jasper K.W. Gerritsen

j.gerritsen@erasmusmc.nl

1 Department of Neurosurgery, Erasmus Medical Center Rotterdam, Dr. Molewaterplein 40, 3015 GD Rotterdam, The Netherlands
Supplement, we can draw the conclusion that we have included 8 studies from the pre-Stupp era. Of those studies, only 3 of them contain overall survival data. The average median survival of these 3 papers combined is 13.6 months (weighted). Two of these papers included patients operated under general anesthesia (GA) without ISM, with an average median survival of 13.5 months (weighted), and one paper included patients operated with the use of ISM, with an average median survival of 16.4 months. When we compare the average median survival data of the papers from the pre-Stupp era with the overall average, we can conclude that this is in line with these survival data and would be unlikely to represent any bias (GA/without ISM 13.5 months pre-Stupp vs. 12.0 months overall; with ISM 16.4 months pre-Stupp vs. 16.9 months overall).

Furthermore, they state that the age of patients is higher in the group treated under GA without ISM in comparison with the group treated with ISM and that this might represent a bias in OS analysis. We agree that age is a strong prognostic factor for e.g. overall survival in GBM patients. Therefore, we further analyzed the data regarding the mean age versus overall survival in our included studies. The average age in the group treated under GA without ISM is 62.4 years (37 studies with data regarding mean age) as compared with 56.9 years in the group treated with ISM ( 9 studies with data regarding mean age). However, this takes into regard all studies, with and without relevant overall survival data. When we analyze the cohort of studies that contain both mean age and overall survival data (both of these parameters we need to draw conclusions regarding differences between groups), we conclude that the mean age in the cohort of studies including patients treated with ISM (3 studies) is actually higher than in the cohort of studies including patients treated under GA without ISM (15 studies) (avg. age 61.2 years in the ISM group as opposed to 60.5 years in the ISM group), which is in contrast with the statement at the beginning of this paragraph and we therefore respectfully disprove it.

Dr. Giussani and Dr. Di Cristofori inquired us to elaborate on some aspects regarding the (added) value and use of $\mathrm{AC}$ in 
GBM surgery. First, they question if there are real benefits for GBM patients to have surgery with AC. We feel fortunate to say that we can state that there are many benefits in using $\mathrm{AC}$ in GBM surgery. In addition to our meta-analysis, we conducted a retrospective matched case-control study in our center in which we matched every patient operated under AC with 3 patients operated under GA (without ISM), including 148 patients in total [1]. We concluded that the extent of resection was significantly higher in the AC group: mean extent of resection in the AC group was $94.89 \%(\mathrm{SD}=10.57)$ as compared with $70.30 \%$ in the GA group $(\mathrm{SD}=28.37)(p=0.0001)$. Furthermore, the mean rate of late minor postoperative complication in the $\mathrm{AC}$ group $(0.03 ; \mathrm{SD}=-0.16)$ was significantly lower than in the GA group $(0.15 ; \mathrm{SD}=0.39)(p=0.05)$, which is in line with our meta-analysis, in which we found that patients operated with ISM experienced less late neurological complications (mean rate 0.13 (95\% CI $0.20-0.23$ ) vs. 0.21 (95\% CI 0.10-0.16); $p<0.001)$. Multiple studies show that the extent of resection improves survival in GBM patients and that patients with gross total resections (GTR) derived the most benefit from the adjuvant therapy [2-5]. However, since $>50 \%$ of GBMs are located in or near eloquent areas [6], there is an increased risk of neurological morbidity when increasing extent of resection. This is in contrast with the fact that due to the limited prognosis of these patients, preservation of quality of life in these patients should be the first concern. AC is able to increase extent of resection in GBM surgery while preserving quality of life, thereby significantly improving patient outcomes. AC could thus be of high value in the surgical treatment of GBM in eloquent areas [7-11].

Secondly, the letter's authors inquired if subcortical mapping is the only way to preserve subcortical white matter tracts during surgery, given the growth pattern of GBMs. Besides from brain mapping (during awake craniotomy) are "asleep" mapping methods another excellent tool to preserve these tracts (MEP, SSEP, continuous dynamic mapping). For example, Prof. Raabe's Neurosurgery Dept. in Bern (Switzerland) uses continuous dynamic mapping with a monopolar for GBM surgeries adjacent to motor eloquent areas [12, 13]. They realize continuous (temporal coverage) and dynamic (spatial coverage) mapping by integrating the mapping probe at the tip of the suction device. Acoustic feedback indicates proximity to the corticospinal tract. New intraoperative developments like these can be combined with ever-improving diagnostics and radiomics (DTI, HARDI, q-ball imaging) to yield optimal results in GBM surgeries in eloquent areas.

Lastly, they pose the question if an accurate microsurgical technique, aimed to circumferentially "peal," can resect the tumor mass en bloc (when possible) instead of internally aspirating it, thereby overpassing the need for AC/ISM in GBM surgery. We would like to stress that GBMs can never be resected radically nor circumferentially "pealed" (like, for example, in meningioma surgery) because ample evidence has shown that GBMs are characterized by leaving behind microscopic tumor satellites into the normal brain parenchyma, deeming tumor recurrences (despite maximal surgical resection followed by adjuvant chemoradiation) unfortunately inevitable [6, 14]. Hence, the very essence of using AC/ISM is to enable the surgeon to determine maximal boundaries for tumor resection while preserving neurological function. As a result, an accurate microsurgical technique like the ones mentioned will fail to overpass the need for techniques in GBM surgery such as AC/ISM.

Ultimately, we agree with Dr. Giussani and Dr. Di Cristofori that GBM surgery would preferably be personalized, taking into account all the patient, tumor, and molecular characteristics. The results of our multicenter RCT will provide vital data and results to enable the neurosurgical community to take the next step in creating a more standardized approach in the (surgical) management of GBMs. Indeed, the results of this trial will give an indication (1) if the use of AC/ISM yields superior outcomes in GBM surgery, but more specifically (2) which GBM patients will reap the most benefit from using AC/ISM.

Moreover and in line with their last statement, we concur that creating an international, multicenter, prospective registry of intraoperative techniques in GBM surgery would be highly appreciated. We would therefore invite the letter's authors to participate in the ENCRAM program. The aim of ENCRAM (European and North-American Consortium and Registry for Awake surgery and intraoperative stimulation Mapping) is to collect, analyze, and report clinical data of patients who have undergone GBM surgery using AC/ISM in an international research collaboration setting.

Research consortia and registries like ENCRAM are next to cost-effective also highly flexible, being able to incorporate numerous (surgical) techniques and parameters, and can be custom-made for each participating center. In this way, combining robust evidence of large-scale trials and registries has the potential to reach (partial) consensus about the (surgical) management of GBMs in the future.

Respectfully,

Jasper K.W. Gerritsen, MD

Arnaud J.P.E. Vincent, MD, PhD

Department of Neurosurgery, Erasmus Medical Center Rotterdam, The Netherlands

\section{Compliance with ethical standards}

Conflict of interest The authors declare that they have no conflict of interest.

\section{References}

1. Gerritsen JK, Viëtor CL, Rizopoulos D et al (2019) Awake craniotomy versus craniotomy under general anesthesia without surgery 
adjuncts for supratentorial glioblastoma in eloquent areas: a retrospective matched case-control study. Acta Neurochir (Wien) 161: 307-315

2. Van den Bent MJ, Stupp R, Mason W (2005) Impact of the extent of resection on overall survival in newly diagnosed glioblastoma after chemo-irradiation with temozolomide: further analyses of the EORTC study 26931. Eur J Cancer Suppl 3:134

3. Smith JS, Cha S, Chang EF et al (2008) Role of extent of resection in the long-term outcome of low-grade hemispheric gliomas. J Clin Oncol 26:1338-1345

4. Stummer W, Meinel T, Pichlmeier U et al (2008) Extent of resection and survival in glioblastoma multiforme: identification of and adjustment for bias. Neurosurgery. 62:564-576

5. Sanai N, Berger MS (2008) Glioma extent of resection and its impact on patient outcome. Neurosurgery. 62:753-764

6. Omuro A, DeAngelis LM (2008) Glioblastoma and other malignant gliomas: a clinical review. JAMA. 310:1842-1850

7. Gupta DK, Chandra PS, Ohja BK et al (2007) Awake craniotomy versus surgery under general anesthesia for resection of intrinsic lesions of eloquent cortex - a prospective randomized study. Clin Neurol Neurosurg 109:335-343

8. Peruzzi P, Bergese SD, Viloria A et al (2011) A retrospective cohort-matched comparison of conscious sedation versus general anesthesia for supratentorial glioma resection. Clinical article. J Neurosurg 114:633-639
9. Sacko O, Lauwers-Cances V, Brauge D et al (2011) Awake craniotomy vs surgery under general anesthesia for resection of supratentorial lesions. Neurosurgery. 68:1992-1998

10. Hervey-Jumper SL, Li J, Lau D et al (2015) Awake craniotomy to maximize glioma resection: methods and technical nuances over a 27-year period. J Neurosurg 123:325-339

11. De Witt Hamer PC, Robles SG, Zwinderman AH et al (2012) Impact of intraoperative stimluation brain mapping on glioma surgery outcome: a meta-analysis. J Clin Oncol 30:2559-2565

12. Schucht P, Seidel K, Beck J et al (2014) Intraoperative monopolar mapping during 5-ALA-guided resections of glioblastomas adjacent to motor eloquent areas: evaluation of resection rates and neurological outcome. Neurosurg Focus 37:E16. https://doi.org/10. $3171 / 2014$

13. Raabe A, Beck J, Schucht P et al (2014) Continuous dynamic mapping of the corticospinal tract during surgery of motor eloquent brain tumors: evaluation of a new method. J Neurosurg 120: 1015-1024

14. Giese A, Bjerkvig R, Berens ME et al (2003) Cost of migration: invasion of malignant gliomas and implications for treatment. J Clin Oncol 21:1624-1636

Publisher's note Springer Nature remains neutral with regard to jurisdictional claims in published maps and institutional affiliations. 